\title{
Effect of parent-delivered action observation therapy on upper limb function in unilateral cerebral palsy: a randomized controlled trial
}

\author{
EMMA KIRKPATRICK ${ }^{1}$ | JANICE PEARSE ${ }^{2}$ | PETER JAMES ${ }^{3}$ | ANNA BASU ${ }^{4}$
}

1 Southampton Clinical Trials Unit, University of Southampton, Southampton; 2 Paediatric Occupational Therapy Services, Newcastle upon Tyne Hospitals NHS Foundation Trust, Newcastle upon Tyne; $\mathbf{3}$ Institute of Health and Society, Newcastle University, Newcastle upon Tyne; $\mathbf{4}$ Institute of Neuroscience, Newcastle University, Newcastle upon Tyne, UK.

Correspondence to Anna Basu, Level 3, Sir James Spence Institute, Royal Victoria Infirmary, Queen Victoria Road, Newcastle upon Tyne NE1 4LP, UK. E-mail: anna.basu@ncl.ac.uk

\section{PUBLICATION DATA}

Accepted for publication 4th February 2016

Published online

\section{ABBREVIATIONS}

AHA Assisting Hand Assessment

MA2 Melbourne Assessment 2

UCP Unilateral cerebral palsy
AIM To determine whether home-based, parent-delivered therapy comprising action observation $(A O)$ and repeated practice $(\mathrm{RP})$ improves upper limb function more than $\mathrm{RP}$ alone in children with unilateral cerebral palsy (UCP).

METHOD Design: single-blinded parallel-group randomized controlled trial with 1:1 allocation comparing AO+RP (intervention) with RP alone (control). Randomization: computergenerated, with allocation concealment by opaque sequentially-numbered envelopes. Setting: northern England, August 2011 to September 2013. Participants: 70 children with UCP; mean age 5.6 years (SD 2.1), 31 female. Intervention: home-based activities were provided, tailored to interests and abilities. Duration: 15 minutes/day, 5 days/week for 3 months. Assessments: Assisting Hand Assessment (AHA; primary outcome measure), Melbourne Assessment 2 (MA2), and ABILHAND-Kids at baseline, 3 months, and 6 months.

RESULTS Outcome data was available at 3 months for 28 children in the AO+RP group and 31 controls, and at 6 months for 26 and 28 children respectively. There were no between-group differences in AHA, MA2, or ABILHAND-Kids at 3 or 6 months versus baseline (all $p>0.05$ ). Combined-group improvements $(p<0.001)$, observed in AHA and MA2 at 3 months, were maintained at 6 months. ABILHAND-Kids also showed improvement at 3 months $(p=0.003)$, maintained at 6 months.

INTERPRETATION Parent-delivered RP (with or without AO) improves upper limb function and could supplement therapist input.
Unilateral cerebral palsy (UCP) is the commonest form of cerebral palsy (CP), with a prevalence of 0.6 per 1000 live births. ${ }^{1}$ Characteristic features of hand function in UCP include weak grasp, reduced speed, loss of fine motor skills, and spasticity. Known effective interventions for the upper limb, such as constraint-induced movement therapy (CIMT) and hand-arm bimanual intensive therapy (HABIT), ${ }^{2}$ are time-intensive and require high levels of therapist input. However, insufficient therapy service provision for children with disabilities is an internationally recognized problem. Other models of service delivery must be explored, including family-centred, parent-delivered approaches to supplement therapist input. One such intervention which lends itself to a parent-delivered approach is action observation (AO) therapy.

The discovery of the Mirror Neuron System, a bilateral cortical network activated similarly during action observation and execution, and contributing to motor learning, ${ }^{3}$ led to investigations of $\mathrm{AO}$ therapy for the upper limb in stroke. ${ }^{4,5}$ The rationale was that AO primed brain regions involved in movement production, enhancing the benefits of subsequent movement practice. ${ }^{6}$ Results in stroke were encouraging, ${ }^{4,7,8}$ but few studies have assessed AO therapy in children with CP. Buccino et al. ${ }^{9}$ undertook a pilot study of 15 children aged 6 to 11 years with hemiplegia or tetraplegia. Children attended 15 physiotherapist-led rehabilitation sessions at a clinical centre. During sessions, both groups watched three to four video clips (which for the intervention group showed motor tasks), each lasting three minutes, and each followed by 2 minutes of therapistguided action execution. Improvement in the Melbourne Assessment of Unilateral Upper Limb Function (MUUL) ${ }^{10}$ occurred in the intervention group only. However, no follow-up data beyond the immediate post-treatment period were provided. Subsequently Sgandurra et al. ${ }^{11}$ studied 24 children aged 6 to 14 years with UCP, using a similar protocol to Buccino. ${ }^{9}$ Children underwent 15 hour-long therapy sessions within 3 weeks. One week post-intervention, the intervention group had improved significantly more than controls on the Assisting Hand Assessment (AHA), ${ }^{12}$ 
but the effect was not sustained. Interestingly, no betweengroup differences were observed in the MUUL. ${ }^{13}$

Intervention delivery in these studies is therapistintensive and, arguably, does not make the best use of therapist time and skills. Recently, parent-delivered occupational therapy home programmes for children with CP were shown to improve performance and parental satisfaction with upper limb function, ${ }^{14}$ and CIMT $^{15}$ and HABIT $^{16}$ have also been explored in home-based settings with encouraging findings. We reasoned that with appropriate training and support, parents could undertake AO therapy with their child in the home, freeing up therapist time for more specialist input. Moreover, rather than viewing videoed movements, children could observe parents modelling the movements, potentially facilitating motor learning. We envisaged a play-based approach to enhance task engagement.

We aimed to assess the effectiveness of parent-delivered play-based AO therapy with repeated practice $(\mathrm{AO}+\mathrm{RP})$ versus $\mathrm{RP}$ alone. We hypothesized that the $\mathrm{AO}+\mathrm{RP}$ group would show greater improvements in hand function than the RP group. We also aimed to assess the overall effectiveness of the home-based play-therapy by combining the $\mathrm{AO}+\mathrm{RP}$ and $\mathrm{RP}$ groups. We predicted an improvement between baseline and 3-month follow-up, maintained at 6 months.

\section{METHOD}

We undertook a single-centre, single-blinded (outcomes assessor) parallel-group randomized controlled trial (RCT) with 1:1 allocation. The trial was registered (ISRCTN 65947097) and approved by Newcastle \& North Tyneside 2 Research Ethics Committee.

\section{Participants and setting}

Children aged 3 to 10 years with UCP predominantly affecting arm and hand function were eligible for inclusion. Children who were registered visually impaired, unable or unwilling to understand or attempt the tasks, or with no active grasp in the affected hand, were excluded, as were children who were expecting another intervention or who had undergone an intervention in the preceding 3 months. Such interventions included upper limb botulinum toxin injections, surgical intervention, or introduction of Lycra sleeves/suits or similar. Recruitment was through clinicians based at 10 hospitals in northern England. All studies took place at the Sir James Spence Institute, Royal Victoria Infirmary, Newcastle upon Tyne.

\section{Interventions}

The intervention was an individualized parent-delivered home-based play therapy programme. AO+RP and RP interventions were both based on repeated movement practice and differed by a single detail: the control (RP) group played independently (with parental supervision), while the $\mathrm{AO}+\mathrm{RP}$ group watched a parent perform the movement each time before attempting it. In both groups, parents sat
What this paper adds

- A parent-delivered home-based action observation therapy programme was developed for unilateral cerebral palsy.

- Improvements in hand function following action observation therapy were similar to those with repeated practice alone.

- Home-based parent-delivered therapy programmes could supplement therapist input and deserve exploration.

next to the child, facing the same direction, and on the side of the less-affected hand. Thus, the AO+RP group could observe parental hand movements from an egocentric viewpoint. Additionally, the parent was on the side of the child least likely to be affected by visual neglect or field defects. Details of instructions for each group are available (Data SI-III, online supporting information, or tiny.cc/ genericinstruction).

Baseline assessments, specifically AHA, ${ }^{12}$ Melbourne Assessment-2 (MA2) ${ }^{17}$ and ABILHAND-Kids,${ }^{13}$ were used to establish areas of difficulty in manual activities for each child, such as reaching, grasp, and manipulating small items. These, along with the child's interests and abilities, informed the choice of intervention activities. Families were provided with materials for the activities along with individually tailored, illustrated instruction booklets emphasizing the desired movements, plus the seating instructions and allocation-specific approach. $\mathrm{AB}$ explained the approach and demonstrated the activities to parents (Data SI-III, online supporting information, or tiny.cc/play-therapy for examples). To reduce cross-contamination, we asked families not to discuss the details of their participation.

The intervention lasted 3 months. Parents were asked to deliver five sessions per week, each lasting 15 minutes. They received a therapy diary to document session details, and reward stickers for the children (Data SI-III, online supporting information, or tiny.cc/therapydiary). To enhance compliance and treatment fidelity, families were telephoned fortnightly for support. $\mathrm{AB}$ also undertook a home visit at 6 weeks, delivering new activities to maintain interest and motivation. Overall, each child received around 12 tailored activities.

\section{Outcomes}

The primary outcome measure was the AHA, ${ }^{12}$ assessing spontaneous use ('performance') of the affected hand in bimanual activities. Secondary outcome measures were the $M A 2^{17}$ measuring unimanual capacity, and the ABILHAND-Kids questionnaire ${ }^{13}$ measuring hand function in activities of daily living. These were completed at baseline, 3 months to assess the effect of the intervention, and 6 months to assess maintenance of effects.

Outcome measures were selected based on published recommendations, ${ }^{18}$ to give a comprehensive overview of performance, ${ }^{19}$ capacity, ${ }^{17}$ and function. ${ }^{13}$ All three have excellent psychometric properties. Furthermore, scores on these assessments do not spontaneously improve over 1 year in children with UCP. ${ }^{20}$ However, the ABILHAND-Kids questionnaire is validated only for children aged 6 to 15 years, so was used only for those aged 6 years and above. 


\section{Sample size}

Sample size was calculated using G*Power (http:// www.gpower.hhu.de; Dusseldorf University). A sample of 58 participants could detect an effect size of 0.75 with $80 \%$ power with alpha 0.05 using a two-tailed test. This sample size choice was pragmatic, based on recruitment feasibility, and reflected the size of retained effects in constraint therapy in young children with UCP using the same primary outcome measure. ${ }^{21}$ A recruitment target of 70 participants was set to compensate for possible attrition.

\section{Randomization and blinding}

The allocation schedule was generated independently by a member of Newcastle Clinical Trials Unit. Sequentially numbered sealed opaque envelopes containing the allocations were used, stored in a locked cabinet to which $\mathrm{AB}$ had access. Once written informed consent had been obtained by EK, participants were randomized by $\mathrm{AB}$. Envelopes were opened in numbered order and contained a card labelled 'treatment' or 'control'. $\mathrm{AB}$ recorded the participant name, code, and randomization date on the card.

The study was single-blinded, with the outcomes assessors (EK and JP) naive to group allocation. Families were told the nature of the intervention for their child, but not whether they were in the intervention $(\mathrm{AO}+\mathrm{RP})$ or control group. At the end of the study, all participants were debriefed through the provision of a letter that explained the two trial arms, which arm their child was in, and preliminary findings.

\section{Statistical methods}

Data were analyzed using SPSS 21.0 (IBM Corp., Armonk, NY, USA). Change scores were calculated by subtracting baseline scores from 3-month and 6-month follow-up scores. Descriptive summary statistics were tabulated for each group at baseline and data checked for normality. Chi-squared tests were used to investigate for bias in missing data according to allocation group, side of hemiplegia, age, or sex. Bonferroni correction was used to adjust for pairwise multiple comparisons.

\section{Primary outcome: Assisting Hand Assessment}

Total scores were converted to logit-based AHA units. ${ }^{19}$ To allow for within-participant correlations over time, we fitted a linear mixed-effects model with a compound symmetry repeated covariance structure, participant specified as a variable with correlated random effects, and time as a repeated measure. Variables were group, time (baseline, 3mo, and $6 \mathrm{mo}$ ), and group*time as fixed effects, with age as a covariate. Estimation of parameters was undertaken using the restricted maximum likelihood method.

\section{Secondary outcome (I): Melbourne Assessment 2}

The MA2 comprises four ordinal subscales (accuracy, fluency, range of movement, and dexterity), each expressed as a percentage. Change scores from 0 to 3 months and 0 to 6 months were compared between groups for each subscale using Mann-Whitney $U$ tests. Where no between-group differences were found, combined-group scores were compared at baseline, 3 months, and 6 months using Friedman's one-way ANOVA. Post-hoc pairwise comparisons were made using Wilcoxon signed-rank tests.

\section{Secondary outcome (II): ABILHAND-Kids}

The ABILHAND-Kids questionnaire was assessed in children aged 6 years and over. In view of the reduced sample size, non-parametric statistics were used (exactly as for MA2).

\section{RESULTS}

Figure 1 summarizes participant flow. Two children had missing baseline data: video-recorded baseline AHA data for one child in the RP group was lost because of a technical problem, and one child from the $\mathrm{AO}+\mathrm{RP}$ group was unwilling to complete the baseline MA2 assessment. Two children did not receive their allocated intervention: one was found to be ineligible after randomization, and the other completed baseline assessments but then repeatedly failed to attend, eventually withdrawing without receiving any intervention. Eleven children (15.6\%) had missing data on either primary or secondary outcome measures at 3 -month follow-up, which falls within acceptable limits. ${ }^{22}$ Recruitment occurred between August 2011 and March 2013, and assessments were completed by September 2013, when the trial finished. There were no adverse events in relation to either the therapy or the assessments.

Participant demographics and results of baseline assessments by allocation are summarized in Table I. Data analysis was by the original assigned group allocation. Participants with missing baseline data or who did not attend the 3-month visit were however excluded from analysis. Comparisons between children with complete follow-up data and those with missing follow-up data on the basis of age, sex, allocation, and side of hemiplegia confirmed that data were missing at random. No imputation was used, as all available data were included in the analyses. Table II summarizes the change in outcome measure scores at 3- and 6month follow-up for the $\mathrm{AO}+\mathrm{RP}$ and $\mathrm{RP}$ groups.

\section{Assisting Hand Assessment}

The linear mixed model showed no significant interaction between group and time $\left(F_{(2,108.3)}=0.32, p=0.72\right)$ - neither was there any significant main effect of group $\left.\left(F_{(1,} 66.1\right)=0.96, p=0.332\right)$ or age $\left(F_{(1,66.1)}=1.64, p=0.21\right)$. However, there was a significant effect of time $\left(F_{(2,108.3)}=11.4, p<0.001\right)$. Pairwise comparisons of the combined groups over time showed significant improvements from baseline to 3 months (95\% confidence interval [CI]: 0.90-2.96 logit-based AHA units, $p<0.001, d=0.55)$, and from baseline to 6 months (95\% CI: 0.46-2.58 logit-based AHA units, $p=0.002, d=0.42$ ). 


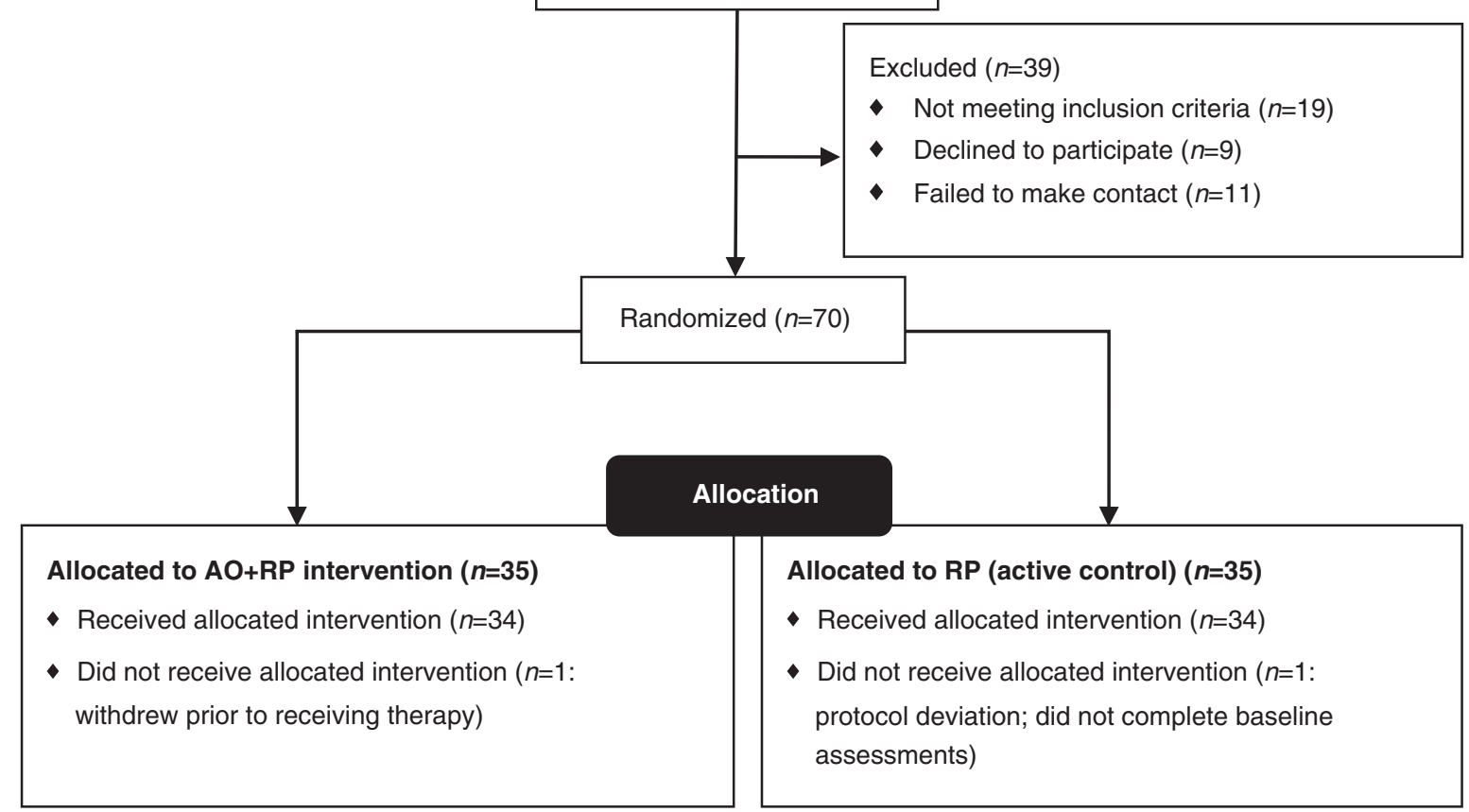

\section{3 mo follow-up}

Followed-up at $3 \mathrm{mo}(n=28)$

Lost to follow-up ( $n=4)$

- Unavailable for follow-up appointment $(n=3)$

- Unable to contact $(n=1)$

Discontinued intervention $(n=2)$

- Lack of engagement $(n=2)$
Followed-up at $3 \mathrm{mo}(n=31)$

Lost to follow-up ( $n=3)$

- Unavailable for follow-up appointment $(n=2)$

- Unable to contact $(n=1)$

Discontinued intervention $(n=0)$

\section{6mo follow-up}

\section{Followed-up at $6 \mathrm{mo}(n=26)$}

Lost to follow-up after 3 mo assessment $(n=2)$

- Unavailable for follow-up appointment $(n=1)$

- Unable to contact $(n=1)$

\section{Followed-up at $6 \mathrm{mo}(n=28)$}

Lost to follow-up after 3 mo assessment $(n=3)$

- Unavailable for follow-up appointment $(n=2)$

- Unwilling to attend follow-up appointment $(n=1)$

\section{Analysis}

\section{Analysed $0-3$ mo data $(n=28)$}

- Including all participants followed-up at 3mo

\section{Analysed $0-6$ mo data $(n=26)$}

- Including all participants followed-up at $6 \mathrm{mo}$
Analysed 0-3mo ( $n=30)$

- Including participants followed-up at 3mo, apart from one participant with missing baseline AHA data due to a technical error

Analysed $0-6$ mo data $(n=27)$

- Including participants followed-up at $6 \mathrm{mo}$, apart from one participant with missing baseline AHA data due to a technical error (as above)

Figure 1: CONSORT diagram indicating participant flow. AO, action observation; RP, repeated practice; AHA, Assisting Hand Assessment. 


\begin{tabular}{|c|c|c|c|}
\hline & & $\mathrm{AO}+\mathrm{RP}$ & $\mathrm{RP}$ \\
\hline Age & Median (IQR) & $\begin{array}{l}5 y 2 \mathrm{mo} \\
(4 \mathrm{y})\end{array}$ & $\begin{array}{l}5 y 4 \mathrm{mo} \\
\text { (3y } 4 \mathrm{mo})\end{array}$ \\
\hline Sex & $n$ Female & 18 & 13 \\
\hline $\begin{array}{l}\text { Side } \\
\text { affected }\end{array}$ & $n$ Left side & 19 & 21 \\
\hline $\mathrm{AHA}$ & $\begin{array}{l}n \text { (missing) } \\
\text { Mean (SD) } \\
\text { (Logit-based } \\
\text { AHA units) }\end{array}$ & $\begin{array}{c}35(0) \\
63.2(16.9)\end{array}$ & $\begin{array}{c}33(2) \\
60.5(15.0)\end{array}$ \\
\hline MA2 & $n$ (missing) & $34(1)$ & $34(1)$ \\
\hline ROM (\%) & Median (IQR) & $68.5(32.2)$ & 61.7 (31.9) \\
\hline $\operatorname{ACC}(\%)$ & Median (IQR) & $83.3(27.2)$ & $82.0(36.3)$ \\
\hline FLU (\%) & Median (IQR) & $61.0(38.1)$ & $52.4(29.9)$ \\
\hline DEX (\%) & Median (IQR) & $59.4(38.4)$ & $50.0(32.3)$ \\
\hline ABILHAND- & $n$ (missing) & $14(0)$ & $13(1)$ \\
\hline Kids $^{a}$ & $\begin{array}{l}\text { Median (IQR) } \\
\text { (logits) }\end{array}$ & $1.48(2.11)$ & $1.03(1.81)$ \\
\hline
\end{tabular}

${ }^{a}$ Based on children aged 6 years and older. $A O$, action observation; $\mathrm{RP}$, repeated practice; IQR, interquartile range. AHA, Assisting Hand Assessment; MA2, Melbourne Assessment 2, subscore abbreviations: ROM, range of movement; ACC, accuracy; FLU, fluency; DEX, dexterity.

\section{Melbourne Assessment 2}

Figure 2 summarizes the MA2 subscale change scores from baseline for each group. No significant between-group differences in change scores were identified on any subscale compared with baseline. With regards to combined group changes, all subscales showed significant improvement at 3 months compared with baseline (Range of motion: $\mathrm{z}=4.61$, $p<0.001, \mathrm{r}=0.42$; Accuracy: $\mathrm{z}=4.09, p<0.001, \mathrm{r}=0.38$; Fluency: $\mathrm{z}=3.02, p=0.003, \mathrm{r}=0.28$; Dexterity: $\mathrm{z}=3.30, p=0.001$, $\mathrm{r}=0.30$ ). These changes persisted at 6 months (Table SI, online supporting information).

\section{ABILHAND-Kids}

There was no difference in improvement on ABILHANDKids scores between $\mathrm{AO}+\mathrm{RP}$ and $\mathrm{RP}$ groups at 3 months $(U=46, p=0.56, r=0.14)$ or 6 months $(U=58, p=0.90$, $r=0.04)$. However, there were significant combined-group changes over time $\left(\chi^{2}=12.5, \mathrm{df}=2, p=0.002\right)$. Pairwise com- parisons showed an increase in scores between baseline and 3 months (median improvement 0.67 logits, $z=-2.73$, $p=0.003, r=0.42)$, persisting at 6 months.

\section{Compliance}

Forty-two therapy diaries were returned (22 from the $\mathrm{AO}+\mathrm{RP}$ group). The mean number of play sessions was 48.2 (SD 19.3) in the therapy group and 54.8 (SD 23.1) in the control group.

\section{DISCUSSION}

Our study did not demonstrate a significant improvement in hand function with $\mathrm{AO}+\mathrm{RP}$ compared with $\mathrm{RP}$ alone in children age 3 to 10 years with UCP. This finding is in contrast to Sgandurra et al. ${ }^{11}$ Key differences between these trials include the setting, dose, and duration of therapy and sample size.

\section{Setting}

Our trial differs from those previously undertaken ${ }^{9,11}$ in using a parent-delivered, home-based therapy model. We chose this model because of the straightforward nature of the approach and the potential to supplement therapy services, which currently cannot provide high-intensity upper limb input to all eligible children. However, monitoring of treatment fidelity is more challenging in this setting than in a clinic-based environment with therapist delivery. Written instructions, training, telephone support, therapy diaries, and a home visit were used to encourage adherence to the allocated intervention. However, although the importance of the watching and copying approach was emphasized in the therapy group, and parents in the control group were told to encourage their child to undertake the activities independently, they could not be strictly monitored. Children in the AO+RP group may not always have observed movements before performing them, because of poor attention or lack of parental emphasis. Thus it is possible that the approaches for the two groups differed less than intended. Our conclusion remains that within a home-based parent-delivered setting, the approaches produce similar outcomes. It is perhaps also

Table II: Change scores from baseline for primary and secondary outcome measures at 3-and 6-month follow-up

\begin{tabular}{|c|c|c|c|c|c|c|}
\hline & & & \multicolumn{2}{|c|}{ 3mo follow-up } & \multicolumn{2}{|c|}{ 6mo follow-up } \\
\hline & & & $\mathrm{AO}+\mathrm{RP}$ & $\mathrm{RP}$ & $\mathrm{AO}+\mathrm{RP}$ & $\mathrm{RP}$ \\
\hline \multicolumn{2}{|c|}{$\mathrm{AHA}$} & $n$ & 28 & 30 & 26 & 27 \\
\hline \multicolumn{2}{|c|}{$\begin{array}{l}\text { Logit-based } \\
\text { AHA units }\end{array}$} & Mean $(95 \% \mathrm{Cl})$ & $2.2(1.3,3.1)$ & $1.6(0.6,2.6)$ & $1.7(0.2,3.3)$ & $1.2(0.4,2.7)$ \\
\hline \multicolumn{2}{|c|}{ MA2 } & $n$ & 28 & 31 & 26 & 27 \\
\hline \multirow[t]{4}{*}{$\%$} & ROM & Mdn (95\% Cl) & $7.4(4.4,10.7)$ & $7.4(3.7,11.8)$ & $3.7(0.0,14.8)$ & $3.7(0.2,13.7)$ \\
\hline & $\mathrm{ACC}$ & Mdn (95\% Cl) & $4.8(1.2,12.0)$ & $5.9(5.0,16.1)$ & $4.7(4.0,12.7)$ & $4.0(0.0,14.7)$ \\
\hline & FLU & Mdn $(95 \% \mathrm{Cl})$ & $2.4(-0.6,9.5)$ & $4.8(2.4,11.9)$ & $2.4(1.4,14.3)$ & $9.5(2.4,14.3)$ \\
\hline & DEX & Mdn $(95 \% \mathrm{Cl})$ & $8.8(3.1,18.8)$ & $0.0(0.0,12.5)$ & $10.1(6.3,18.8)$ & $6.7(3.1,15.6)$ \\
\hline \multirow{2}{*}{\multicolumn{2}{|c|}{$\begin{array}{l}\text { ABILHAND- Kids } \\
\text { Logits }\end{array}$}} & $n$ & 11 & 10 & 11 & 11 \\
\hline & & $\operatorname{Mdn}(95 \% \mathrm{Cl})$ & $0.67(0.2,-1.7)$ & $0.67(-0.4,1.4)$ & $0.50(-0.9,1.7)$ & $0.74(-0.5,1.4)$ \\
\hline
\end{tabular}

AO, action observation; RP, repeated practice; AHA, Assisting Hand Assessment; MA2, Melbourne Assessment 2; Mdn, median score; ROM, range of movement; ACC, accuracy; FLU, fluency; DEX, dexterity. Results are shown in bold where $95 \%$ Cl (confidence interval) for change scores do not overlap 0. 

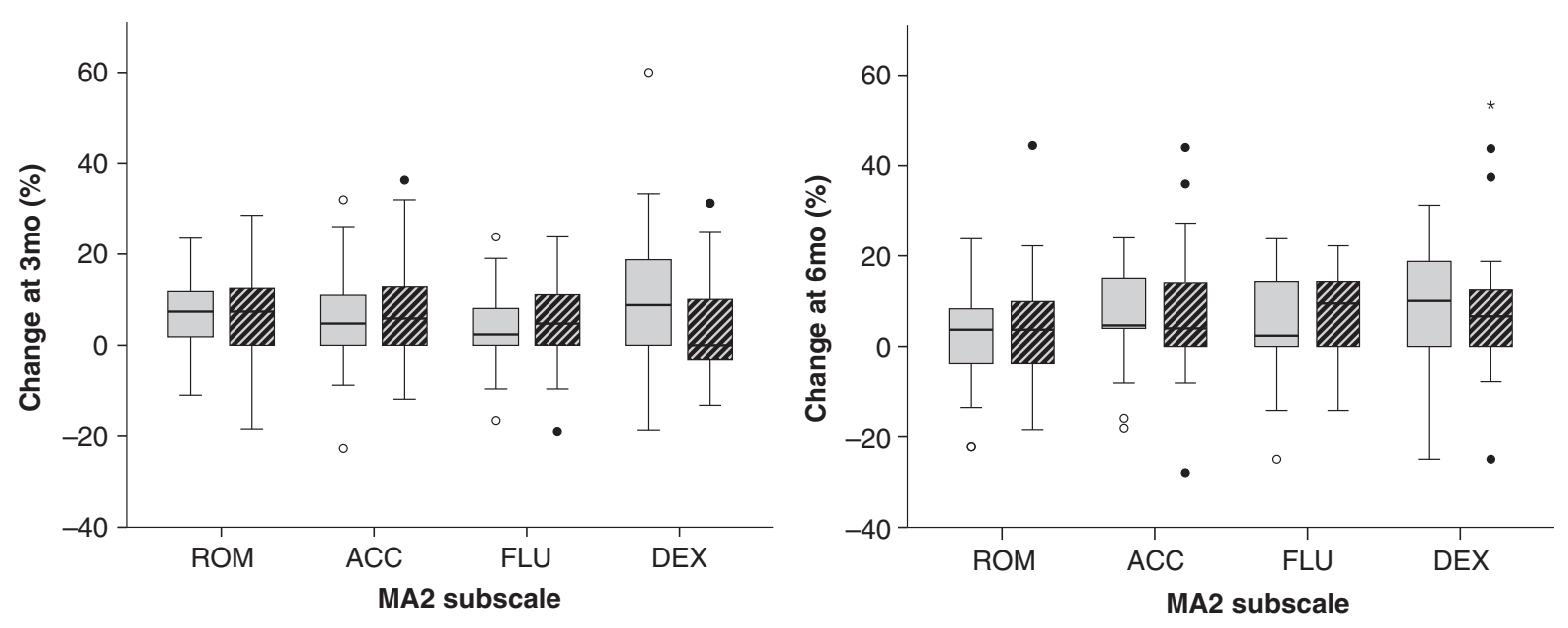

Figure 2: Box and whisker plots summarizing change in Melbourne Assessment 2 (MA2) subscale scores. Left: from baseline to 3 months. Right: from baseline to 6 months. Solid grey bars: AO+RP; striped bars: RP. AO, action observation; RP, repeated practice; ROM, range of movement; ACC, accuracy; FLU, fluency; DEX, dexterity.

surprising that the control group from Sgandurra et al. ${ }^{11}$ did not show benefits from repeated practice.

\section{Dose and duration}

In our trial, participants were asked to undertake activities for 15 minutes, five times per week for 3 months. Compliance data showed that $62 \%$ of the children who returned therapy diaries achieved this dose, while $78 \%$ achieved or exceeded 1 hour per week of therapy. The optimal dose for upper limb therapy has not been defined. Novak et al. ${ }^{14}$ demonstrated improvements on the Quality of Upper Extremity Skills Test following 4 weeks of a home programme with 4.5 hours of intervention. We chose a brief session duration to accommodate the short attention spans of younger children and to produce a potentially sustainable approach. We aimed to provide around 15 hours of therapy - though small compared with doses used in intensive approaches such as CIMT and HABIT, it is comparable to the dose used by Sgandurra et al. ${ }^{11}$ It would be interesting to determine the effects of a longer period of intervention or a higher dose. For future studies we would also advocate documentation of dose at more frequent intervals to enhance compliance data.

Another aspect of dose is the number of movement repetitions practised. Children in the RP group may have practised movements with greater frequency than the AO+RP group, who alternated observation and performance. Addressing this limitation is difficult - if we had prescribed double the therapy time to the $\mathrm{AO}+\mathrm{RP}$ group as to the $\mathrm{RP}$ group, time engaged in therapy would have been a confounding factor. Similarly, if we had made the RP group observe unrelated actions or non-human motion between moves, we would have risked unblinding and possibly alienating parents, as this would not represent a plausible component of therapy. Pragmatically, if our results repre- sent a trade-off between repetitions and time, this does not provide strong justification for advocating $\mathrm{AO}+\mathrm{RP}$ over $\mathrm{RP}$ alone.

\section{Sample size}

Our sample size was large compared with many studies of interventions for children with UCP. The mean sample size for RCTs of upper limb therapies in UCP is 29.7 (SD 19.1). ${ }^{23}$ Our power calculation was based on a standardized effect size of 0.75 . A post-hoc power calculation based on our collected data showed that 322 participants would be needed to detect a between-group change difference of five logit-based AHA units (smallest detectable difference) with $80 \%$ power. No published trials of interventions for children with UCP have achieved samples of this size.

\section{Combined group improvements}

We showed that a low-intensity parent-delivered home programme of play therapy improves hand function in children with UCP. The improvement in AHA scores was smaller than the smallest detectable difference. ${ }^{19}$ However, not all changes in bimanual function are detectable with the AHA. Hung et al. ${ }^{24}$ detected group-specific improvements in bimanual coordination using 3D kinematic recordings from a drawer-opening task, which were not observed using the AHA. The MA2 is a reformulation of the MUUL with improved psychometric properties; however, standard error of measurement and smallest detectable difference have not yet been defined. ${ }^{17}$ Nonetheless, small but significant improvements were observed in the combined group data in all three outcome measures used.

Our approach is accessible and the findings generalizable within the age group studied. However, it does require 
parental time and commitment, and engagement from both parties.

A potential weakness is that we did not have an additional group receiving no intervention for 6 months, but it would have been difficult to recruit to such a group in the context of the trial. We considered adding a further baseline test to demonstrate lack of change in the 3 months preceding the intervention, but this would have increased attrition. However, MUUL and AHA scores are not significantly different when measured 12 months apart in children with UCP. ${ }^{20}$ Even in young children with UCP (around 3y of age), the average developmental increase in AHA scores over a 3 -month period is $<0.5$ logits. ${ }^{25}$ Furthermore, the improvements in our study were seen in the first 3 months, with no significant improvement between 3- and 6-month assessments. Therefore our results likely indicate genuine therapeutic gains rather than age-related changes. Of interest for future studies is the effect of age in response to therapy intervention in each group. Unfortunately our sample size was not large enough to analyze this. It will also be important to document the intensity of any other therapy input received.

\section{CONCLUSION}

Using a home-based parent-delivered play therapy approach, there is no difference in upper limb outcomes between $\mathrm{AO}+\mathrm{RP}$ therapy and $\mathrm{RP}$ alone for children aged 3 to 10 years with UCP. However, low-intensity upper limb therapy can be delivered at home by parents, incorporated into play for as little as 1 hour per week with a small but sustained benefit.

\section{ACKNOWLEDGEMENTS}

Funding: WellChild Trust, Newcastle University (Henry Miller PhD studentship to EK), and NIHR Research Capability Fund. The funding bodies had no role in the design, collection, analysis, or interpretation of this study, nor in manuscript preparation or publication decisions. Dr Anna Basu is funded through a National Institute of Health Research (NIHR) Career Development Fellowship, and was funded through an NIHR Clinical Trials Fellowship and Clinical Lectureship (NIHR-matched scheme) while conducting this trial. The views expressed in this publication are those of the authors and not necessarily those of the NHS, the National Institute for Health Research, or the Department of Health. Thanks to Professor Janet Eyre for helpful preliminary discussions.

\section{SUPPORTING INFORMATION}

The following additional material may be found online:

Table SI: Statistical comparison of changes in MA2 sub scores. Data SI: Example game instructions.

Data SII: Generic instructions for control and therapy groups. Data SIII: Therapy diary.

\section{REFERENCES}

1. Johnson A. Prevalence and characteristics of children with cerebral palsy in Europe. Dev Med Child Neurol 2002; 44: 633-40.

2. Charles J, Gordon AM. Development of hand-arm bimanual intensive training (HABIT) for improving bimanual coordination in children with hemiplegic cerebral palsy. Dev Med Child Neurol 2006; 48: 931-36.

3. Rizzolatti G, Craighero L. The mirror-neuron system. Annu Rev Neurosci 2004; 27: 169-92.

4. Ertelt D, Small S, Solodkin A, et al. Action observation has a positive impact on rehabilitation of motor deficits after stroke. Neurolmage 2007; 36: T164-73.

5. Franceschini M, Ceravolo MG, Agosti M, et al. Clinical relevance of action observation in upper-limb stroke rehabilitation: a possible role in recovery of functional dexterity. A randomized clinical trial. Neurorehabil Neural Repair 2012; 26: 456-62.

6. Pomeroy V, Aglioti SM, Mark VW, et al. Neurological principles and rehabilitation of action disorders: rehabilitation interventions. Neurorehabil Neural Repair 2011; 25: $33 \mathrm{~S}-43 \mathrm{~S}$.

7. Celnik P, Webster B, Glasser DM, Cohen LG. Effects of action observation on physical training after stroke. Stroke 2008; 39: 1814-20.

8. Sugg K, Muller S, Winstein C, Hathorn D, Dempsey A. Does action observation training with immediate physical practice improve hemiparetic upper-limb function in chronic stroke? Neurorebabil Neural Repair 2015; 29: 807-17.

9. Buccino G, Arisi D, Gough P, et al. Improving upper limb motor functions through action observation treatment: a pilot study in children with cerebral palsy. Dev Med Child Neurol 2012; 54: 822-28.

10. Randall M, Carlin JB, Chondros P, Reddihough D. Reliability of the Melbourne assessment of unilateral upper limb function. Dev Med Child Neurol 2001; 43: 761-67.

11. Sgandurra G, Ferrari A, Cossu G, Guzzetta A, Fogassi L, Cioni G. Randomized trial of observation and execution of upper extremity actions versus action alone in children with unilateral cerebral palsy. Neurorehabil Neural Repair 2013; 27: 808-15.

12. Krumlinde-Sundholm L, Holmefur M, Kottorp A, Eliasson AC. The Assisting Hand Assessment: current evidence of validity, reliability, and responsiveness to change. Dev Med Child Neurol 2007; 49: 259-64.

13. Arnould C, Penta M, Renders A, Thonnard JL. ABILHAND-Kids: a measure of manual ability in children with cerebral palsy. Neurology 2004; 63: 1045-52.

14. Novak I, Cusick A, Lannin N. Occupational therapy home programs for cerebral palsy: double-blind, randomized, controlled trial. Pediatrics 2009; 124: e606-14.

15. Chen YP, Pope S, Tyler D, Warren GL. Effectiveness of constraint-induced movement therapy on upper-extremity function in children with cerebral palsy: a system- atic review and meta-analysis of randomized controlled trials. Clin Rebabil 2014; 28: 939-53.

16. Ferre CL, Brandao MB, Hung YC, Carmel JB, Gordon AM. Feasibility of caregiver-directed home-based handarm bimanual intensive training: a brief report. Dev Neurorehabil 2015; 18: 69-74.

17. Randall M, Imms C, Carey LM, Pallant JF. Rasch analysis of The Melbourne Assessment of Unilateral Upper Limb Function. Dev Med Child Neurol 2014; 56: 665-72.

18. Klingels K, Jaspers E, Van de Winckel A, De Cock P, Molenaers G, Feys H. A systematic review of arm activity measures for children with hemiplegic cerebral palsy. Clin Rehabil 2010; 24: 887-900.

19. Krumlinde-Sundholm L. Reporting outcomes of the Assisting Hand Assessment: what scale should be used? Dev Med Child Neurol 2012; 54: 807-08.

20. Klingels K, Feys H, De Wit L, et al. Arm and hand function in children with unilateral cerebral palsy: a one-year follow-up study. Eur 7 Paediatr Neurol 2012; 16: $257-65$.

21. Eliasson AC, Krumlinde-Sundholm L, Shaw K, Wang C. Effects of constraint-induced movement therapy in young children with hemiplegic cerebral palsy: an adapted model. Dev Med Child Neurol 2005; 47: 266-75.

22. Schulz KF, Grimes DA. Sample size slippages in randomised trials: exclusions and the lost and wayward. Lancet 2002; 359: 781-85. 
23. Sakzewski L, Ziviani J, Boyd RN. Efficacy of upper limb therapies for unilateral cerebral palsy: a meta-analysis. Pediatrics 2014; 133: e175-204.

24. Hung YC, Casertano L, Hillman A, Gordon AM. The effect of intensive bimanual training on coordination of the hands in children with congenital hemiplegia. Res Dev Disabil 2011; 32: 2724-31.

25. Holmefur M, Krumlinde-Sundholm L, Bergstrom J, Eliasson AC. Longitudinal development of hand func- tion in children with unilateral cerebral palsy. Dev Med Child Neurol 2010; 52: 352-57. 